IMA Journal of Applied Mathematics (1985) 35, 159-174

\title{
The Formation of Freckles in Binary Alloys
}

\author{
A. C. FOWLER \\ Mathematical Institute, 24-29 St Giles, Oxford, OX1 3LB
}

[Received 19 June 1985 and in revised form 7 August 1985]

\begin{abstract}
This paper presents a synopsis of some recent work, still in progress, aimed at elucidating a quantitative explanation of the processes by which flow chimneys form when certain types of alloys are directionally solidified. If (for example) light fluid is released at the liquid-solid "mushy" (dendrite) zone, and cooling is from below, then the intermediate fluid flow undergoes convection through the porous dendrite mass. This can lead to an "instability" of the form of the mushy zone, such that upwelling light fluid flows preferentially in channels within the dendrite mass. What we seek to develop here, is a mathematical basis by which this phenomenon may be properly understood. Accordingly, a mathematical model is developed, simplified, and partially analysed, and as a result we are able to make one specific prediction concerning a criterion for the onset of convection and freckling. This prediction is equivalent to the classical Rayleigh number condition for convective instability.
\end{abstract}

\section{Introduction}

WHEN A MULTT-COMPONENT LIQUID is solidified, it is very often the case that a planar solid-liquid interface is highly (morphologically) unstable, and that the actual interface is a highly irregular surface, which takes the form of small dendrite arms occupying a zone of finite thickness, often called the mushy zone. The reason for this fact is that the solution for a planar interface leads to "constitutional" supercooling, wherein the liquid ahead of the interface has a temperature below the liquidus, despite being above the interfacial temperature, because of the dependence of the liquidus (freezing) temperature on solute concentration (Flemings, 1974). The common occurrence of dendritic zones is due to the fact that component diffusivities are invariably much smaller than thermal diffusivities, so that the thickness of the region over which the concentration changes in the liquid is much thinner than the corresponding region over which the temperature changes, and one can think of the dendrite zone as performing the function of smearing out the concentration jump over a much larger distance than in the planar case.

If light fluid is released in a mushy zone being cooled from below (or heavy fluid is released when cooling from above), the possibility exists that convection of the intermediate fluid will occur due to compositional buoyancy effects. In this case, a type of flow instability is known to exist, which results in the formation of various types of compositional segregation, and in particular to the formation of "freckles" (Mehrabian, Keane \& Flemings, 1970a, b; Copley, Giamei, Johnson \& Hornbecker, 1970; McDonald \& Hunt, 1969, 1970). Systems in which such segregation occurs are, for example, solidification of $\mathrm{Ni}-\mathrm{Al}$ and $\mathrm{Al}-\mathrm{Cu}$ alloys, and crystallization of 
$\mathrm{NH}_{4} \mathrm{Cl}-\mathrm{H}_{2} \mathrm{O}$ solution. The latter, in particular, is especially suitable for laboratory demonstration.

Freckles are the manifestation of flow pipes within the mushy zone, i.e. regions of zero solid fraction, and are known to arise due to a kind of instability if the convective flow velocity within the mushy zone becomes too large (Flemings, 1974). Specifically, if the component of the fluid flow in the growth direction exceeds the isotherm velocity, then the fluid must warm up as it moves through the dendrites. To maintain equilibrium, dendrites are melted; but if the mass fraction of solid is locally decreased, then flow resistance is also decreased, so that flow there increases, leading to further melting, and so on. Thus, the accepted metallurgical criterion for the formation of freckles is that

$$
d T / d t>0,
$$

where $T$ is temperature, and $d / d t$ is a material derivative following the fluid in the mush. One purpose of this paper is to provide a mathematical framework in which to examine the criterion (1.1). This framework may perhaps stand in relation to the criterion (1.1), as the Mullins-Sekerka (1964) morphological stability analysis does to the constitutional supercooling explanation of cellular solidification.

There has been much interest in materials science in the prediction of channeltype segregation. By the nature of the subject, this has been directed towards solution of practical problems involving more complicated systems (Kou et al., 1978; Szekely \& Jassal, 1978; Ridder, Kou \& Mehrabian, 1981; Fujii, Poirier \& Flemings, 1979), but one could argue that the more fundamental aspects of the problem have been inadequately treated, having taken for granted various apparently arbitrary assumptions: for example, that the liquid fraction in the mush depends only on temperature (Mehrabian et al., 1970a), or that the temperature in the mush varies linearly with depth (Maples \& Poirier, 1984).

The purpose of the present paper is complementary to that adopted in the metallurgical literature. We will consider the simplest possible configuration in which we may study the solidification phenomenon, but will include as many physical processes as might be thought reasonable. In particular, no arbitrary assumptions will be made. Instead, we use the well-tried techniques of nondimensionalization and scaling to reduce the equations of the complete system to those which contain the essential physics, but which are as simple as possible. These can then be used, in principle, to understand various features of freckle formation.

The study of the equations describing two-phase (mushy) flows at the freezing point has recently attracted much interest in geophysical contexts; two examples are melt generation and flow processes in partially-molten rocks (McKenzie, 1984) (flow at the melting-point) and the study of the solidification of the earth's inner core (Jacobs, 1953). This latter process involves the release of the light component (possible sulphur or oxygen) in the outer-core iron alloy, to form the iron innercore. It is an obvious and likely possibility that the compositional convection induced by this process is what drives the geodynamo. In pursuit of this objective, Loper, Roberts, and co-workers have been led to formulate very general models for the solidification of a binary alloy involving a slurry-like mushy layer (this would also presumably be appropriate for crystallization in magma chambers (McBirney 
\& Noyes, 1979), where nucleation sites abound) (Loper \& Roberts, 1978, 1980), and then, more recently, for a dendritically crystallizing binary alloy (Hills, Loper \& Roberts, 1983). A simplified version of this model was used to study the probable nature of a dendritic mush, when flow chimneys are present (Roberts \& Loper, 1983). The specific situation addressed was that of cooling from below, typified by the experiment on ammonium chloride by Copley et al. (1970). The authors did not attempt to enquire how the chimneys came into being, and so the object of the present paper is, to a certain extent, to address this problem. In addition, the mathematical approach adopted is somewhat different. More recently Huppert \& Worster (1985) have presented a more phenomenological model, which was successfully compared to experiment. In its present state, this model ignores interdendritic convection, and so is not suited to the present purpose.

The rest of this paper is organized as follows: in Section 2 we present a reasonably complete mathematical model. This is essentially contained in Hills et al.'s (1983) thermodynamically consistent model, but may be more immediately accessible. In Section 3 the problem is non-dimensionalized and scaled, and a steady state solution is given. In Section 4, the model is reduced by making various simplifications, notably of zero solidification shrinkage, zero partition coefficient, and infinite Lewis number, in a way suggested by (and consistent with) the steady solution, and this leads to a free-boundary problem in the mush involving three coupled non-linear partial differential equations. We identify a Rayleigh number $R$ for the problem, and show that convection is initiated at a finite critical value of $R$, which may be explicitly computed in the "weakly non-eutectic" limit, and we examine the criterion for chimney formation (1.1) in this context. Conclusions and suggestions follow in Section 6.

\section{Mathematical Model}

The model presented here is essentially that of Hills et al. (1983), and accordingly is presented as briefly as possible. We consider the mush to be a homogeneous two phase medium at local thermodynamic equilibrium. Define the "solute" to be the light component (of a binary alloy), and let $\xi^{s}$ and $\xi^{l}$ be solidus and liquidus concentrations (measuring the respective weight fractions), and let $\phi$ be the mass fraction of solid phase. Then the mixture concentration may be defined as

$$
\xi=\phi \xi^{s}+(1-\phi) \xi^{l}
$$

The density is defined as

$$
\rho=\rho^{s}+\rho^{l},
$$

where

$$
\rho^{s}=\phi \rho, \quad \rho^{l}=\rho(1-\phi),
$$

these being respectively mass per unit volume of mixture of solid and liquid; they are related to the actual solid and liquid densities $\rho_{s}$ and $\rho_{l}$ by

$$
\rho^{l}=\rho_{l} \alpha, \quad \rho^{s}=(1-\alpha) \rho_{s},
$$


where $\alpha$ is the void fraction (liquid volume fraction). Conservation of mass in the mush yields

$$
\begin{aligned}
& \frac{\partial \rho^{s}}{\partial t}+\nabla \cdot\left[\rho^{s} \mathbf{u}^{\mathbf{t}}\right]=m^{s}, \\
& \frac{\partial \rho^{l}}{\partial t}+\nabla \cdot\left[\rho^{l} \mathbf{u}^{\mathrm{l}}\right]=m^{l}=-m^{s},
\end{aligned}
$$

where $u^{s}, \mathbf{u}^{l}$ are solid and liquid velocities, and $m^{l}$ is the local rate of melting. To be specific, we will assume

$$
\mathbf{u}^{s}=\mathbf{0},
$$

as roughly appropriate to dendritic growth (barring dendrite erosion and breakage). The barycentric velocity $u$ is defined as

$$
\mathbf{u}=\phi \mathbf{u}^{3}+(1-\phi) \mathbf{u}^{l}=\chi^{\mathbf{v}},
$$

where we define the liquid mass fraction and liquid velocity,

$$
\chi=1-\phi, \quad \mathbf{v}=\mathbf{u}^{l} .
$$

Conservation of species can be written in the form

$$
\frac{\partial}{\partial t}(\rho \xi)+\nabla \cdot\left[\rho^{s} \mathbf{u}^{s} \xi^{s}+\rho^{l} \mathbf{u}^{l} \xi^{l}\right]=\nabla \cdot\left[\rho^{l} D \nabla \xi^{l}\right],
$$

which simplifies to

$$
\rho \frac{d \xi}{d t}+\nabla \cdot\left[\rho \chi(1-\chi)\left(\xi^{l}-\xi^{\xi}\right) \mathbf{v}\right]=\nabla \cdot\left[\rho \chi D \nabla \xi^{l}\right] .
$$

In this equation we have neglected solid state diffusion. The equation for $\xi^{s}$ can thus be written as

$$
\frac{\partial}{\partial t}\left(\rho^{s} \xi\right)=d^{s}
$$

where we have used (2.6), and defined the volumetric deposition rate $d^{3}$. If we assume that liquid concentrations are microscopically uniform (this is equivalent to the approximation which underlies the Scheil equation), then it is appropriate to define

$$
d_{s}=\lambda \xi^{l} m^{s},
$$

where the partition coefficient $\lambda$ is the ratio of solidus and liquidus slopes, and will be assumed constant. We assume Darcy's law for a porous medium

$$
\nabla=-\kappa \chi\left[\nabla p-\rho_{l} \mathbf{B}\right]
$$

where $\kappa$ is related to the usual definition of the permeability $K$ via

$$
\kappa=K / \eta_{1} \alpha \chi,
$$

and $\eta_{l}$ is the liquid viscosity. 
We will assume the thermal conductivities of solid and liquid are equal (as is reasonable for $\mathrm{NH}_{4} \mathrm{Cl}-\mathrm{H}_{2} \mathrm{O}$ and metallic alloys, though not for some other aqueous solutions). Then the energy equation (neglecting the work done by pressure changes, as appropriate for industrial processes) is

$$
L m^{l}+\rho c_{p} \frac{d T}{d t}=k \nabla^{2} T+E,
$$

where $L$ is the latent heat, $c_{p}$ is the specific heat and the interfacial energy source (due to the viscous heat released by the shearing of the microscale flow) is

$$
E=\frac{\rho_{s}}{\kappa \rho_{l}} \frac{|y|^{2}}{(1-\chi)} \text {. }
$$

It is included for completeness, but will be neglected henceforth as being small in laboratory contexts.

We suppose that the liquidus is the linear relationship

$$
T=T_{0}-\Gamma \xi^{l},
$$

where $\Gamma$ is the liquidus slope, this being satisfied everywhere in the mush, assuming local thermodynamic equilibrium. Further, we suppose

$$
\begin{aligned}
& \rho_{s}=\rho_{s}^{0}\left[1-\alpha_{s}\left(T-T_{0}\right)-\beta_{s} \xi\right], \\
& \rho_{l}=\rho_{l}^{0}\left[1-\alpha_{l}\left(T-T_{0}\right)-\beta_{l} \xi\right],
\end{aligned}
$$

where $\alpha_{s}, \alpha_{l}$ are solid and liquid thermal expansion coefficients, and $\beta_{s}, \beta_{l}$ are corresponding compositional coefficients; this completes the specification of the mush equations.

Boundary conditions follow from the integral forms of the various conservation laws. If $V$ is the interface velocity (either solid/mush or liquid mush), then jump conditions are

$$
\begin{aligned}
\mathbf{V} \cdot \mathbf{n}[\rho]_{-}^{+} & =[\rho \chi \mathbf{v} \cdot \mathbf{n}]_{-}^{+}, \quad \text { (mass) } \\
\mathbf{v} \cdot \mathbf{n}[\rho \xi]_{-}^{+} & =\left[\left\{\rho \chi \mathbf{v} \xi^{\prime}-\rho \chi D \nabla \xi^{l}\right\} \cdot \mathbf{n}\right]_{-}^{+}, \quad \text { (species) } \\
\xi^{l} & =c_{E}, \\
T & \left.=T_{E},\right\} \text { (if at eutectic) } \\
{[T]_{-}^{+} } & =0, \quad \text { (thermodynamic) } \\
\mathbf{V} \cdot \mathbf{n}\left[\rho c_{p} T+\rho \chi L\right]_{-}^{+} & =\left[\left\{\rho c_{p} T \mathbf{u}+\rho L \chi \mathbf{v}-k \nabla T\right\} . n\right]_{ \pm}^{+} \text {(energy) }
\end{aligned}
$$

At the mush/liquid boundary, we specify

$$
\xi^{s}=\lambda \xi^{\prime}, \quad\left[\xi^{\prime}\right]_{-}^{+}=0, \quad[p]_{-}^{+}=0
$$

the first of these is the usual jump condition at a solid/liquid interface, and the second assumes that the temperature is uniform on the dendritic micro-scale, which will be the case providing solute diffusion is large enough, compared to the microscopic length scale, to make the concentration locally uniform, and providing we neglect surface energy; the third is continuity of stress, supposing that deviatoric stresses are much smaller than hydrostatic pressure variations. In addition, some 
definition of how the mush/liquid boundary is defined is necessary. One such is that

$$
\chi=1 \text { on mush/liquid boundary. }
$$

Another is that the dendrite tips move such that they are marginally stable to constitutional supercooling (Langer, 1980). This means that the actual temperature gradient in the liquid at the mush/liquid interface should equal the gradient of the liquidus temperature (in the liquid) there. A little manipulation of (2.20) and (2.23), using (2.19), (2.22), $\left[\xi^{l}\right]_{-}^{ \pm}=0$, and (2.17), shows that $\left[D \partial \xi^{l} / \partial n\right]^{ \pm} \leqslant 0$ and $[k \partial T / \partial n]_{-}^{+} \leqslant 0$, only if $(2.25)$ holds. If we suppose $D$ and $k$ are constant then it can be deduced from (2.17) that the condition of (marginal) stability at the tips is equivalent to (2.25), though this need not be so if $D$ and $k$ are different in solid and liquid. We will adopt (2.25) henceforward.

To summarize the equations, there are essentially six, expressing conservation of mass, energy, momentum (Darcy), solid and liquid concentrations and equation of state. Using the constitutive relations (2.17), (2.16), these are

$$
\begin{gathered}
-\rho c_{p} \Gamma \frac{d c}{d t}-L \frac{\partial}{\partial t}[\rho(1-\chi)]=-k \Gamma \nabla^{2} c, \\
\rho \frac{d}{d t}[\chi c+(1-\chi) s]+\nabla \cdot[\rho \chi(1-\chi)(c-s) v]=\nabla \cdot[\rho \chi D \nabla c], \\
\frac{\partial}{\partial t}[\rho(1-\chi) s]=\lambda c \frac{\partial}{\partial t}[\rho(1-\chi)], \\
\nabla=-\kappa \chi\left[\nabla \dot{p}+\rho_{l} g \mathrm{j}\right], \\
\rho=\rho_{s}(1+r \chi)^{-1},
\end{gathered}
$$

where

$$
r=\left(\rho_{\mathrm{s}}-\rho_{\mathrm{l}}\right) / \rho_{\mathrm{l}}
$$

we have written

$$
\xi^{\prime}=c, \quad \xi=s,
$$

$\mathbf{j}$ is a unit upwards vector, and

$$
\rho_{t}+\nabla \cdot[\rho \chi v]=0 .
$$

We now proceed to a non-dimensionalization of this set. It should be added that separate equations describing the variables in the single phase solid and liquid regions are necessary. Since these are more familiar, their presentation is postponed until the following section.

\section{Non-dimensionalization and Basic Steady State}

The logical procedure is to seek a steady (basic) solution, and then use this to suggest typical scales. However, for clarity of presentation it is more convenient to non-dimensionalize first. We suppose that a solidification rate $V=|\mathbf{V}|$ is prescribed. In practice, this is itself determined by the heat flow in the solid, but we will suppose that it is given: this corresponds industrially to the process of zone melting. 
Roughly speaking, we choose a thermal length scale $\kappa_{T} / V$, and thermal time scale $\kappa_{T} / V^{2}$, where $\kappa_{T}$ is the thermal diffusivity

$$
\kappa_{T}=k / \rho_{s} c_{p}
$$

This is because the mush thickness is controlled by thermal processes rather than compositional diffusivity, as mentioned in Section 1. Specifically, put

$$
\begin{gathered}
\mathbf{x}=\gamma \kappa_{T} \mathbf{x}^{*} / V, \quad \mathbf{v}=V \mathbf{v}^{*}, \quad t=\gamma \kappa_{T} t^{*} / V^{2}, \quad \rho=\rho_{s} \rho^{*}, \\
p=p_{0}-\rho_{l}^{0} g y+\gamma \kappa_{T} p^{*} / \kappa, \quad T=T_{0}+\Gamma T^{*},
\end{gathered}
$$

where the inverse Stefan number $\gamma$ is given by

$$
\gamma=c_{p} \Gamma / L \lesssim O(1)
$$

With these substitutions, the equations $(2.26)-(2.33)$ become (dropping asterisks on the dimensionless variables)

$$
\begin{gathered}
\rho=(1+r \chi)^{-1}, \\
\rho_{\mathbf{t}}+\nabla \cdot[\rho \mathbf{u}]=0, \\
\rho \frac{d}{d t}[\chi c+(1-\chi) s]+\nabla \cdot[\rho(1-\chi)(c-s) \mathbf{u}]=\frac{1}{\gamma L \mathbf{e}} \nabla \cdot[\rho \chi \nabla c], \\
\frac{\partial}{\partial t}[\rho(1-\chi) s]=\lambda c \frac{\partial}{\partial t}[\rho(1-\chi)], \\
\gamma \rho \frac{d c}{d t}+\frac{\partial}{\partial t}[\rho(1-\chi)]=\nabla^{2} c, \\
\left.\mathbf{u}=\chi^{2}[-\nabla p+R c]\right],
\end{gathered}
$$

where

$$
\begin{gathered}
R=\frac{\Delta \rho g \kappa}{V}, \quad \Delta \rho=\left(\beta_{1}-\alpha_{l} \Gamma\right) \rho_{l}^{0}, \\
\text { Le }=\kappa_{T} / D .
\end{gathered}
$$

$R$ is the Rayleigh number, Le is the Lewis number, and $\mathbf{u}$ is the barycentric velocity.

These equations are supplemented by those in the solid and liquid regions. In the solid,

$$
\gamma T_{t}=\nabla^{2} T
$$

governs the temperature. In the liquid, the dimensionless Boussinesq equations become, with the above scaling,

$$
\begin{gathered}
\nabla \cdot \mathbf{u}=0, \\
\gamma\left[T_{t}+\mathbf{u} \cdot \nabla T\right]=\nabla^{2} T, \\
\gamma\left[c_{t}+\mathbf{u} \cdot \nabla c\right]=\frac{1}{\mathrm{Le}} \nabla^{2} c, \\
\left.\frac{v \gamma}{\sigma} \frac{d \mathbf{u}}{d t}=-\nabla p+v \nabla^{2} \mathbf{u}+\left(R_{\alpha} T+R_{\beta} c\right)\right],
\end{gathered}
$$


where

$$
\begin{aligned}
R_{a} & =\alpha_{l} \Gamma \rho_{l}^{0} g \kappa / V, \\
R_{b} & =\beta_{l} \rho_{l}^{0} g \kappa / V, \quad R=R_{b}-R_{a}, \\
\sigma & =\eta_{l} / \rho_{l} \kappa_{T}, \\
v & =\eta_{l} \kappa V^{2} / \kappa_{T}^{2} \gamma^{2} .
\end{aligned}
$$

$\sigma$ is the Prandtl number. The permeability coefficient $\kappa$, defined in (2.13) and (2.14), can be related to a typical dendrite arm spacing length $l$ by the relation (Nye \& Frank, 1973)

$$
\kappa=l^{2} / X \eta_{l},
$$

where $\eta_{l}$ is the liquid viscosity, and $X$ is a numerical factor of order $10^{2}$ or $10^{3}$. Thus $\kappa$ incorporates the microscale $l$. It is instructive to rewrite the parameters $v$ and $R$ in terms of the macroscopic length scale $h$, defined from (3.2) as

$$
h=\gamma \kappa_{T} / V .
$$

Using (3.11) to eliminate $V$ from the definitions of $v$ and $R$ in (3.9) and (3.5), we find

and

$$
v=l^{2} / X h^{2}
$$

$$
R=\frac{\Delta \rho g h^{3}}{\kappa_{T} \eta_{t}} \frac{v}{\gamma}
$$

Typical laboratory values might be $l \sim 1 \mathrm{~mm}, h \sim 1 \mathrm{~cm}$, so $v \sim 10^{-5}$ is very small. Also (in the laboratory) $h \sim 1 \mathrm{~cm}, g \sim 10^{3} \mathrm{~cm} \mathrm{~s}^{-2}, \Delta \rho \sim 1 \mathrm{gm} \mathrm{cm}^{-3}$, $\kappa_{T} \sim 10^{-2} \mathrm{~cm}^{2} \mathrm{~s}^{-1}, \eta_{l} \sim 10^{-2} \mathrm{gm} \mathrm{cm}^{-1} \mathrm{~s}^{-1}$, so that the usual fluid Rayleigh number is $\sim 10^{7}$. With $\gamma=1$, this gives a dendritic Rayleigh number $R \sim 10^{2}$.

The boundary conditions are the following: as $z$ (the vertical co-ordinate) $\rightarrow \infty$ in the liquid,

$$
T \rightarrow \Delta, \quad c \rightarrow c_{\infty},
$$

where $\Delta+c_{\infty}>0$ so that the liquid is above the freezing point. We also would require some flow conditions, which will be omitted for the moment, as the liquid flow problem will be of secondary importance.

On the liquid/mush interface, (2.19)-(2.25) imply

$$
\begin{gathered}
{[T]_{-}^{+}=[c]_{-}^{+}=[p]_{-}^{+}=0,} \\
\chi=1, \quad s=\lambda c, \\
{[p]_{-}^{+}=[\mathbf{u} \cdot \mathbf{n}]_{-}^{+}=0,} \\
{\left[\frac{\partial T}{\partial n}\right]_{-}^{+}=\left[\frac{\partial c}{\partial n}\right]_{-}^{+}=0 .}
\end{gathered}
$$

On the mush/solid boundary, we similarly find

$$
\begin{aligned}
\text { V.n }[\rho]_{-}^{+} & =[\rho \mathbf{u} \cdot \mathbf{n}]_{-}^{+}, \\
\text {V. } \mathbf{n}[\rho(1-\chi)]_{-}^{+} & =\left[\frac{\partial T}{\partial n}\right]_{-}^{+},
\end{aligned}
$$


or

$$
-\rho s^{-} \mathbf{V} \cdot \mathbf{n}+\mathbf{V} \cdot \mathbf{n}[\rho \chi c+\rho(1-\chi) s]=\left[\rho c u-\frac{1}{\gamma \operatorname{Le}} \rho \chi \nabla c\right] \cdot \mathrm{n}^{+}
$$

$$
c=c_{E}, \quad T=T_{E} .
$$

In both (3.15) and (3.16), $\mathbf{V}$ is the dimensionless velocity of the respective interface. In the solid/mush condition, the first alternative $(3.16)_{3}$ only occurs if the composition is above eutectic. For simplicity, we will suppose this interface is at the eutectic temperature and composition.

Additionally, we would require $T$ prescribed as $z \rightarrow-\infty$, but not if the solidification rate $V$ is prescribed.

\subsection{Steady Solution}

The system (3.4), (3.8), (3.14)-(3.16) is complicated and it will help to make some judicious simplifications. The first is what we shall call the extended Boussinesq approximation, which simply neglects solidification shrinkage, i.e.

$$
r=0 \text {. }
$$

This is quite reasonable for metallic alloys, and is a very convenient simplification.

Then $\rho=1$ in the mush, and (3.4) become

$$
\begin{gathered}
\nabla \cdot \mathbf{u}=0, \\
\mathbf{u}=-\chi^{2}[\nabla p-R c j], \\
\frac{d}{d t}[\chi c+(1-\chi) s]+\nabla \cdot[(1-\chi)(c-s) \mathbf{u}]=\frac{1}{\gamma L e} \nabla \cdot[\chi \nabla c], \\
\frac{\partial}{\partial t}[(1-\chi) s]=-\lambda c \frac{\partial \chi}{\partial t}, \quad \gamma \frac{d c}{d t}-\frac{\partial \chi}{\partial t}=\nabla^{2} c .
\end{gathered}
$$

Our strategy now is the following. We wish to build up a theory of convection in the mush, and to do so, we follow the classical approach to Rayleigh-Bénard convection. Thus we will first study the steady situation in which $u=0$, and then examine its stability to convective perturbations.

Let us suppose that the interfacial velocities of the mush are constant, $V=1$. Working downwards from the liquid zone, the describing equations and boundary conditions are then

as $z \rightarrow \infty, T \rightarrow \Delta, c \rightarrow c_{\infty}$;

in $z>z_{l}$,

$$
\begin{aligned}
& \gamma T_{\mathrm{t}}=\nabla^{2} T, \\
& \gamma c_{\mathrm{t}}=\frac{1}{\mathrm{Le}} \nabla^{2} c
\end{aligned}
$$

on $z=z_{l}$,

$$
\begin{gathered}
{[T]_{-}^{+}=[c]_{-}^{+}=\left[\frac{\partial T}{\partial n}\right]_{-}^{+}=\left[\frac{\partial c}{\partial n}\right]_{-}^{+}=0,} \\
\chi=1, \quad s=\lambda c ;
\end{gathered}
$$




$$
\begin{aligned}
\text { in } z_{l}>z>z_{m}, & \frac{\partial}{\partial t}[\chi c+(1-\chi) s]=\frac{1}{\gamma L e} \nabla \cdot[\chi \nabla c], \\
\frac{\partial}{\partial t}[(1-\chi) s] & =-\lambda c \frac{\partial \chi}{\partial t}, \quad \gamma \frac{\partial c}{\partial t}-\frac{\partial \chi}{\partial t}=\nabla^{2} c,
\end{aligned}
$$

on $z=z_{m}$,

$$
1-\chi^{+}=\left[\frac{\partial T}{\partial n}\right]_{-}^{+}, \quad c=c_{E}, \quad T=T_{E} .
$$

We look for a solution which depends only on the vertical co-ordinate $y=z-t$, and without loss of generality we put $y=0$ on $z=z_{m}$; then if $y=y_{l}$ is the mush/liquid boundary, $y_{1}$ is to be determined. The solutions for $y>y_{1}$ are

$$
\begin{aligned}
& T=\Delta-A e^{-\gamma\left(y-y_{i}\right),} \\
& c=c_{\infty}+B e^{-\gamma L e\left(y-y_{i}\right)},
\end{aligned}
$$

where $A, B$ are as yet arbitrary. In the mush, $c$ and $\chi$ satisfy

$$
\begin{aligned}
-[\chi c+(1-\chi) s]^{\prime}= & \frac{1}{\gamma \operatorname{Le}}\left[\chi c^{\prime}\right]^{\prime}, \quad[(1-\chi) s]^{\prime}=-\lambda c \chi^{\prime}, \\
& -\gamma c^{\prime}+\chi^{\prime}=c^{\prime \prime},
\end{aligned}
$$

and satisfaction of the boundary conditions yields

and

$$
A=\left(\Delta+c_{\infty}\right) /(1-1 / \mathrm{Le}), \quad B=A / \mathrm{Le}
$$

$$
s=\lambda\left(c_{\infty}+B\right), \quad \chi=1, \quad c=c_{\infty}+B, \quad c^{\prime}=-\gamma A \quad \text { on } y=y_{1} .
$$

First integrals of (3.21) with (3.23) yield

$$
\begin{gathered}
\chi=c^{\prime}+\gamma c+1+\gamma \Delta, \\
\chi c+(1-\chi) s=c_{\infty}-\frac{1}{\gamma \operatorname{Le}} \chi c^{\prime},
\end{gathered}
$$

and the boundary conditions to be specified by $c$ are

$$
y=0, \quad c=c_{E} ; \quad y=y_{l}, \quad c=c_{\infty}+B .
$$

Since (3.24) and $(3.21)_{2}$ give a pair of first-order differential equations for $c$ and $s$, the extra condition in (3.25) determines $y_{1}$, the mush thickness. We now assume that $\gamma$ Le $\gg 1$, as is likely to be true in practice. (3.24) is a quadratic equation for $c^{\prime}$, with two solutions $c^{\prime} \sim O(1), c^{\prime} \sim O\left(\gamma\right.$ Le). However, if $c^{\prime} \gg 1$, then $\chi \sim c^{\prime}$ from (3.24), which is clearly absurd, since $\chi \sim 1$. It follows that the appropriate solution is $c^{\prime} \sim O(1)$. Neglecting $O(1 / \gamma \mathrm{Le}$ ), and for simplicity taking $\lambda \rightarrow 0$ (as appropriate for aqueous solutions), we find that $s=O(\lambda)$, and so

$$
\begin{aligned}
\chi & \sim c_{\infty} / c, \\
c^{\prime} & \sim c_{\infty} / c-\gamma c-(1+\gamma \Delta),
\end{aligned}
$$


with

$$
c \sim c_{\infty} \text { on } y_{i} \quad c=c_{E} \text { on } y=0 .
$$

Thus the mush thickness is given by

$$
y_{1}=\int_{c_{x}}^{c_{t}} \frac{c d c}{\left[\gamma c^{2}+(1+\gamma \Delta) c-c_{\infty}\right]} .
$$

For $\gamma \sim O(1)$, this is $\sim O(1)$, confirming that our choice of length scale was appropriate. As $y$ becomes small (with $\lambda=0), y_{l} \sim O(\ln (1 / \gamma)$ ). Values in the range $0.01-0.1$ are typical for aqueous solutions although for $\mathrm{NH}_{4} \mathrm{Cl}-\mathrm{H}_{2} \mathrm{O}, \gamma \sim 6$ (latent heat is "small"); in this case $y_{l} \sim O(1 / \gamma)$. Typically $\gamma \geq 1$ for metal alloys.

One can also examine a similarity solution of the equations, with no flow (Worster, 1983,1985 ). The similarity form of the equation is no longer integrable (being nonautonomous), but the controlling influence of thermal diffusion in dictating the length scale is still felt. The steady solution reproduced here was given by Hills et al. (1983).

\section{A Reduced Model}

We have already set $r=0$ in Section 3 , and at the end, $\lambda=0$. We wish to consider $\mathbf{u} \neq \mathbf{0}$. In addition to these two approximations, we now recognize the impotence of solutal diffusion by putting $1 / \mathrm{L} e=0$. We are primarily concerned with convection in the dendrite zone, and to this end we will assume that motion in the liquid is purely vertical, and steady:

$$
u=(0,-w(x)),
$$

where henceforth we will concentrate on two-dimensional flows for convenience. Notice that the assumption of a purely vertical flow in the liquid is actually inconsistent with the momentum equation

$$
\frac{v y}{\sigma} \frac{d \mathbf{u}}{d t}=-\nabla p+v \nabla^{2} \mathbf{u}+\left(R_{\alpha} T+R_{\beta} c\right) \mathbf{j}
$$

Since $c \approx c_{\infty}, v \ll 1$ and $R_{a} \ll R$ if $\alpha_{l} \Gamma \ll \beta_{l}$, it is reasonable to suppose that $p \approx 0$ in the liquid. In a stably stratified (cold at the bottom) situation it is also reasonable that the flow in the liquid is entirely due to the compositional convection in the mushy zone. Nevertheless, satisfaction of (4.2) involves further boundary layer analysis of the liquid flow to find the velocity field. In this first simple approach, this problem is not addressed further. In a frame moving upwards with speed $v=1$, if $y$ is the fixed-frame vertical co-ordinate, put

$$
y=z-t
$$

as in Section 3. With the velocity field given by (4.1), exact solutions of (3.8) with the boundary conditions (3.14) and (3.15) and adoption of $1 / \mathrm{Le} \rightarrow 0$, lead to the 
boundary conditions for $c$ on $y_{1}$ - (the mush/liquid boundary):

$$
\begin{aligned}
c & \approx c_{\infty}, \\
\frac{\partial c}{\partial y} & \approx-\gamma(\Delta+c)(w(x)+1) .
\end{aligned}
$$

Notice that we require $w+1>0$ for these solutions to exist, which is precisely the metallurgical condition (of (1.1)) that freckles do not form (at least at the surface). In two dimensions, we adopt a stream function $\psi$ :

thus

$$
\mathbf{u}=\left(\psi_{y},-\psi_{x}\right)
$$

$$
p_{x}=-\psi_{y} / \chi^{2}, \quad p_{y}=R c+\psi_{x} / \chi^{2},
$$

and so (3.4) simplifies to

$$
\begin{aligned}
\nabla \cdot\left[\frac{1}{\chi^{2}} \nabla \psi\right] & =-R c_{x}, \\
\psi, c_{x}-\psi_{x} c_{y} & =\frac{\partial}{\partial y}(\chi c), \\
\frac{\partial}{\partial y}[\chi-\gamma(1-\chi) c] & =\nabla^{2} c,
\end{aligned}
$$

in the mush. Boundary conditions are (4.4) and others from (3.15) and (3.16):

$$
\begin{aligned}
c & =c_{\infty} \quad \text { on } y=y_{l}, \\
\frac{\partial c}{\partial y} & =-\gamma\left(\Delta+c_{\infty}\right)[w(x)+1] \quad \text { on } y=y_{1}, \\
\chi & =1 \quad \text { on } y=y_{l}, \\
c & =c_{E} \quad \text { on } y=y_{s}, \\
\psi & =0 \quad \text { on } y=y_{s} .
\end{aligned}
$$

The two flow conditions, that pressure is continuous and normal velocity is continuous, lead to the exact boundary conditions (in two dimensions)

$$
\begin{aligned}
-\psi_{x} & =\frac{W-U y_{1}^{\prime}}{1+y_{l}^{\prime 2}} \\
\psi_{x} & =y_{1}^{\prime} \psi_{x} \quad(\partial \psi / \partial n=0),
\end{aligned}
$$

where $(U, W)$ are the pure liquid velocity components, and we have assumed (see discussion above) that $p=p_{0}+R_{B} c_{\infty}+$ smaller terms in the liquid. Thus, with $U=0, W=-w$, we have the boundary condition for $\psi$,

$$
\frac{\partial \psi}{\partial n}=0=\psi_{y}-y_{i}^{\prime} \psi_{x} \text { on } y=y_{t}
$$

and the definition of $w$

$$
w(x)=\left(1+y_{i}^{\prime 2}\right) \psi_{x}, \quad y=y_{l}
$$


The problem is to solve (4.7) subject to (4.8), (4.10) and (4.11). There are five conditions for the elliptic equations (4.6) $)_{1,3}$, and one for the first-order equation $(4.6)_{2}$. Since $y_{l}$ is an unknown boundary, this is apparently the right number.

\section{Hypotheses: Onset of Convection, Onset of Freckling}

The set (4.7) is a non-linear set of equations ultimately requiring a numerical solution. In the remainder of this paper, we offer some analytic clues as to the possible behaviour of the solutions. Firstly, let us see how the physical criterion (1.1) relates to the equations (4.7). We write $(4.7)_{2}$ in the form

$$
\left(\mathbf{u}-\mathbf{V}_{l}\right) \cdot \nabla c=-\frac{\partial}{\partial y}[(1-\chi) c]
$$

where $V_{l}=(0,1)$ is the interface velocity. Since $\chi=1$ on $y_{l}$, we require $\partial / \partial y[(1-\chi) c] \leqslant 0$ at $y_{l}$ for a physical $(\chi \leqslant 1)$ solution to exist. That is, $\left(\mathbf{u}-\mathbf{V}_{1}\right) \cdot \nabla c \geqslant 0$. Since $T=-c$, this is $\mathbf{u} . \nabla T \leqslant \mathbf{V}_{l} . \nabla T$, or $d T / d t \leqslant d T / d t_{l}$, where $d / d t$ is the material derivative following the fluid, $d / d t_{1}$ is the derivative following $y_{l}$. But $T=-c_{\infty}$ at $y_{l}$, so $d T / d t_{l}=0$. Thus a physical solution only exists if $d T /\left.d t\right|_{y_{l}} \leqslant 0$, in keeping with (1.1).

\subsection{Convection}

Since $R$ is a Rayleigh number, it is plausible to suppose that for $R<R_{c}$, the basic state $\left(\chi c \approx c_{\infty}, \psi=0\right.$, as in Section 3$)$ is linearly stable, but that for $R>R_{\mathrm{c}}$ convection is initiated with $\|\psi\| \rightarrow 0$ as $R \rightarrow R_{c}$. Proper examination of this awaits a numerical solution, but some useful ideas can be gained by looking at the situation when $c_{\mathcal{E}}-c_{\infty} \ll 1$, i.e. the initial liquid composition is "close" to eutectic. We put

$$
\begin{gathered}
c_{E}-c_{\infty}=\delta \ll 1, \quad \bar{R}=R \delta, \\
c=c_{\infty}+\delta \bar{c}, \\
\chi=1-\delta \bar{\chi}, \quad \\
\psi=\delta \bar{\psi}, \quad x=\delta \bar{x}, \quad y=\delta \bar{y} ;
\end{gathered}
$$

neglecting terms of relative $O(\delta)$, and omitting overbars, the equations (4.7) become

$$
\begin{aligned}
-\nabla^{2} \psi & \sim \bar{R} c_{x}, \\
\psi_{y} c_{x}-\psi_{x} c_{y} & \sim \frac{\partial}{\partial y}\left[c-c_{\infty} \chi\right], \\
\nabla^{2} c & \sim 0,
\end{aligned}
$$

with boundary conditions:

$$
\begin{gathered}
c=1, \quad \psi=0 \quad \text { on } y=0 ; \\
c=0, \quad \chi=0, \quad \partial \psi / \partial n=0, \\
c_{y}=-\gamma\left(\Delta+c_{\infty}\right)\left[1+\left(1+y_{l}^{\prime 2}\right) \psi_{x}\right] \quad \text { on } y=y_{l} .
\end{gathered}
$$


The basic state is then

$$
\psi=0, \quad c=1-b_{0} y, \quad y_{1}=y_{0}=1 / b_{0}=1 / \gamma\left(\Delta+c_{\infty}\right) .
$$

$(5.3)_{2}$ determines $\chi$, but is uncoupled from the other equations so that $(5.3)_{1,3}$ may be studied separately. Linear stability is easily studied (assuming an exchange of stability); the linearized solutions are

$$
\begin{aligned}
c & =1-b_{0} y+A \sinh \alpha y \cos \alpha x, \\
y_{1} & =y_{0}+B \cos \alpha x, \\
\psi & =[C y \cosh \alpha y+D \sinh \alpha y] \sin \alpha x,
\end{aligned}
$$

where $A, B, C, D$ are various constants, and the assumption of marginal stability yields the neutral curve

where

$$
\vec{R}=\frac{4 \theta \cosh ^{2} \theta}{\sinh 2 \theta-2 \theta}
$$

$$
\theta=\alpha y_{0}
$$

The minimum value of $\vec{R}$ is at

$$
\bar{R}_{c} \approx 4.70 \text { when } \theta=1.64,
$$

so that the marginally stable cells have an aspect ratio $\pi / \alpha y_{0} \approx 2$.

In terms of the original $R$, the critical value is

$$
R_{c} \sim 4 \cdot 7 / \delta=4 \cdot 7 /\left(c_{E}-c_{\infty}\right) ;
$$

for $c_{E}-c_{\infty} \approx 0 \cdot 1$, this is $R_{c} \approx 50$. Our previous estimate of $R \sim 10^{2}$ is compatible with this figure.

For moderate values of $\psi$, non-linear stability theory will yield a maximum upwards velocity at $x=0, y=y_{l}$ of the form

$$
v \approx C\left|\vec{R}-\vec{R}_{c}\right|^{t} .
$$

A crucial question is whether the bifurcation is supercritical, as for ordinary convection, or subcritical, as for (diffusive) double-diffusive convection (Proctor, 1981; Da Costa, Knobloch \& Weiss, 1981). This point has not been resolved yet, even for (5.3). If the bifurcation is supercritical, then it is safe to predict that freckle formation is initiated at a value

$$
\bar{R}=\bar{R}_{f} \approx \bar{R}_{c}+1 / C^{2} \sim O(1),
$$

and this should be experimentally verifiable. However, if the bifurcation is subcritical, then it is reasonable that a non-linear disturbance of finite amplitude (of order $\left(\bar{R}_{c}-\bar{R}\right)^{ \pm}$will be able to trigger freckling at values below $\bar{R}_{c}$.

In either event, the model's lateral boundary conditions must be adjusted, once freckling occurs. For a freckle at $x=0$, the boundary conditions on $\psi$ and $c$ must be found by local solution of the Poiseuille-type flow in the chimney. This has not been done, but will provide a means for providing solutions in the freckling régime. 
In concluding this section, let us summarize our results by way of hypothesis:

(i) a dendritically crystallizing binary alloy has (at least at constant solidification velocity) a well-defined Rayleigh number (3.5), or (3.13);

(ii) there exists a critical $R_{c} \sim O(1)$, such that for $0<R<R_{c}$, the steady state in which $\mathbf{u}=\mathbf{0}$ is linearly stable. If $c_{E}-c_{\infty} \ll 1$, an approximation to $R_{c}$ is provided by (5.10), which could possibly serve as the basis for an experimental test;

(iii) there exists a further critical $R_{f}$, such that for values of $R>R_{f}$, freckling is possible. If the bifurcation is supercritical, then $R_{f}>R_{c}$, and freckles do not exist for $R<R_{f}$; the transition is "soft". If the bifurcation is subcritical, then $R_{f} \geqslant 0$, and (roughly) for $R<R_{c}$, freckling will occur provided disturbances to the steady state of $O\left(\left(R_{c}-R\right)^{\ddagger}\right)$ are made. When freckles do exist, extension of the boundary conditions along the lines of Roberts \& Loper (1983) is required, but is not pursued here.

It is obvious that further validation of the above hypothesis requires a fuller analysis, in particular numerical results and a non-linear stability analysis need to be obtained. Neither of these has at present been done, and we offer the present results in the spirit of likely, but not fully verified, possibilities.

\section{Conclusions and Suggestions}

A model can be formulated for the description of a mushy zone in a solidifying binary alloy. With certain simplifications, notably $\mathrm{Le} \rightarrow \infty$, this can be reduced to a set of three non-linear partial differential equations governing the stream function $\psi$, the liquid composition $c$, and the liquid mass fraction $\chi$. The principal parameter controlling the dynamics of the flow is a Rayleigh number

$$
R=\Delta \rho g \kappa / V
$$

where $V$ is solidification rate, and $\kappa$ is related to permeability (e.g. $\kappa=l^{2} / X \eta_{l}$, $l=$ dendrite spacing, $X \sim 10^{3}, \eta_{l}=$ liquid viscosity).

Various kinds of analysis, performed to a greater or lesser degree, suggest the following: for $R<R_{c}$, the static state of no interdendritic fluid flow is stable. For $R>R_{c}$, it is unstable, and steady convection occurs through the mush. For $R>R_{f}>R_{c}$ (if the bifurcation is supercritical), this solution ceases to exist as it stands, and we expect thin flow pipes to occur in the mush. These can be thought of as analogous to thin uprising plumes, but a complete analysis incorporating them has not yet been attempted. If the bifurcation is subcritical, then $R_{f}<R_{c}$, and the instability requires finite excitation for $R<R_{c}$.

The conclusions and hypothesis presented here are to an extent, speculative; further analytical and numerical work remains to be done to bolster the conclusions. However, it seems that a reasonable basis for experimental and numerical verification has been put forward.

I am grateful to Grae Worster for a number of illuminating discussions, also to editor and referees for their suggestions for improving the manuscript. 


\section{REFERENCES}

Copley, S. M., Giamel, A. F., Johnson, S. M. \& Hornbecker, M. F. 1970 The origin of freckles in unidirectionally solidified castings. Metall. Trans. 1, 2193-2204.

DA Costa, L. N., KNOBLOCH, E. \& Weiss, N. O. 1981 Oscillations in double-diffusive convection. J. Fluid Mech. 109, 25-43.

Flemings, M. C. 1974 Solidification Processing. New York: McGraw-Hill.

Flemings, M. C. \& Nereo, G. E. 1967 Macrosegregation: Part I. Trans. metall. Soc. AIME $329,1449-1461$.

FUII, T., PoIrIER, D. R. \& FLEMINGS, M. C. 1979 Macrosegregation in a multi-component low alloy steel. Metall. Trans. 10B, 331-339.

HILLS, R. N., LOPER, D. E. \& ROBERTS, P. H. 1983 A thermodynamically consistent model of a mushy zone. $Q$. J. Mech. appl. Mech. 36, 505-539.

HUPPERT, H. E. \& WORSTER, M. G. 1985 Dynamic solidification of a binary melt. Nature 314, 703-707.

JiCoBs, J. A. 1953 The earth's inner core. Nature 172, 297-298.

Kou, S., PoIrIER, D. R. \& Flemings, M. C. 1978 Macrosegregation in rotated remelted ingots. Metall. Trans. 9B, 711-719.

LANGER, J. S. 1980 Instabilities and pattern formation in crystal growth. Rev. Mod. Phys. 52, $1-28$.

LOPER, D. E. \& ROBERTS, P. H. 1978 On the motion of an iron-alloy core containing a slurry. I. General Theory. Geophys. Astrophys. Fluid Dyn. 9, 289-321.

LOPER, D. E. \& ROBERTS, P. H. 1980 On the motion of an iron-alloy core containing a slurry. II. A simple model. Geophys. Astrophys. Fluid Dyn. 16, 83-127.

MAPLes, A. L. \& PoIRIER, D. R. 1984 Convection in the two-phase zone of solidifying alloys. Metall. Trans. 15B, 163-172.

MCBIRNEY, A. L. \& NoYes, R. M. 1979 Crystallisation and layering of the Skaergaard intrusion. J. Petrology 20, 498-554.

MCDonAlD, R. J. \& HUNT, J. D. 1969 Fluid motions through the partially solid regions of a casting and its importance in understanding A type segregation. Trans. metall. Soc. AIME 245, 1993-1997.

MCDONald, R. J. \& HUNT, J. D. 1970 Convective fluid motion within the interdendritic liquid of a casting. Metall. Trans. 1, 1787-1788.

MCKENZIE, D. P. 1984 The generation and compaction of partially molten rock. J. Petrol. 25, 713-765.

Mehrabian, R., Keane, M. A. \& Flemings, M. C. $1970 a$ Interdendritic fluid flow and macrosegregation; influence of gravity. Metall. Trans. 1, 1209-1220.

Mehrabian, R., Keane, M. A. \& Flemings, M. C. $1970 b$ Experiments on macrosegregation and freckle formation. Metall. Trans. 1, 3238-3241...

Mullins, W. W. \& SEKERKA, R. F. 1964 Stability of a planar interface during solidification of a dilute binary alloy. J. appl. Phys. 35, 444-451.

NYE, J. F. \& FrANK, F. C. 1973 Hydrology of the intergranular veins in a temperate glacier. Association Internationale d'Hydrologie Scientifique. Symposium on the Hydrology of Glaciers, Cambridge, 1969, pp. 157-161.

ProctoR, M. R. E. 1981 Steady subcritical thermohaline convection. J. Fluid Mech. 105, 507-521.

RidDer, S. D., Kou, S. \& Mehrabian, R. 1981 Effect of fluid flow on macrosegregation in axi-symmetric ingots. Metall. Trans. 12B, $435-447$.

ROBERTS, P. H. \& LOPER, D. E. 1983 Towards a theory of the structure and evolution of a dendrite layer. In Stellar and Planetary Magnetism, (Soward, A. M., Ed.), pp. 329-349. New York: Gordon and Breach.

SZEKELY, J. \& JASSEL, A. S. 1978 An experimental and analytical study of the solidification of a binary dendritic system. Metall. Trans. 9B, 389-398.

Worster, M. G. 1983 Convective flow problems in geological fluid mechanics. Ph.D. thesis, Cambridge University (DAMTP).

WORSTER, M. G. 1985 Solidification of an alloy from a cooled boundary. Preprint. 